\title{
Transplantation and Re-transplantation of Mouse Kidney Grafts to Study Local Immune Responses
}

\section{Daqiang Zhao}

3Institute of Organ Transplantation, Tongji Hospital, Tongji Medical College, Huazhong University of Science and Technology, Wuhan, Hubei 430030, China.

\section{Jiangqiao Zhou}

Department of Organ Transplantation, Renmin Hospital of Wuhan University, Wuhan, Hubei 430060, China.

\section{Adham Abu Ali}

Department of Surgery, University of Pittsburgh, School of Medicine, Pittsburgh, PA 15261, USA.

\section{Roger Tieu}

Medical Scientist Training Program, University of Pittsburgh, School of Medicine, Pittsburgh, PA 15261, USA.

\section{Fadi G. Lakkis}

Thomas E. Starzl Transplantation Institute, University of Pittsburgh, School of Medicine, Pittsburgh, PA 15261, USA.

\section{Martin H. Oberbarnscheidt}

Thomas E. Starzl Transplantation Institute, University of Pittsburgh, School of Medicine, Pittsburgh, PA 15261, USA.

\section{Amit Tevar}

Department of Surgery, University of Pittsburgh, School of Medicine, Pittsburgh, PA 15261, USA.

\section{Zhishui Chen}

Institute of Organ Transplantation, Tongji Hospital, Tongji Medical College, Huazhong University of Science and Technology, Wuhan, Hubei 430030, China.

\section{Khodor I. Abou-Daya ( $\sim$ kha17@pitt.edu )}

Thomas E. Starzl Transplantation Institute, University of Pittsburgh, School of Medicine, Pittsburgh, PA 15261 , USA.

\section{Method Article}

Keywords: mouse, kidney transplantation, kidney re-transplantation, immunology, microsurgery.

Posted Date: November 16th, 2021

DOI: https://doi.org/10.21203/rs.3.pex-1679/v1 
License: (c) (i) This work is licensed under a Creative Commons Attribution 4.0 International License. Read Full License 


\section{Abstract}

Mouse kidney transplantation is widely used to study the immune response to allogeneic grafts. This response includes a circulating systemic compartment and a resident non circulating one. A distinction between these compartments remains an important caveat to the interpretation of the resident or local immune response's importance and function. Here, we describe re-transplantation as a method to functionally test the resident component of the primary immune response while also studying the secondary recipient's response. Our detailed, stepwise protocol can be reliably replicated for both the primary and secondary, donor and recipient operations. The techniques in this protocol can be efficiently implemented by an individual proficient in mouse kidney transplantation surgical procedures.

\section{Introduction}

\section{Development and applications of the protocol}

Organ transplantation is a lifesaving treatment for patients with end-stage organ failure. Mouse kidney transplantation models have been widely used as an important investigational approach to study rejection ${ }^{1,2}$. Since the first mouse kidney transplantation experimental model was established in $1973^{3}$, the techniques have been refined and new techniques were developed to improve the survival rate of recipients $4,5,6,7,8,9,10,11,12,13,14$. In our lab, we continued to improve and utilize this model to study innate and adaptive mechanisms of rejection ${ }^{15,16,17,18,19,20}$. In the past three decades, significant advances have been made in prevention of acute graft rejection and improving early graft survival. However, chronic graft rejection remains a major obstacle to long-term allograft survival ${ }^{21}$. It is therefore necessary to further our understanding of the pathophysiology of chronic rejection.

Graft rejection is caused by host immune response to non-self ${ }^{22,23}$. This response includes a circulating systemic compartment and a resident non circulating one. The resident compartment is maintained locally within the graft and most likely contributes to chronic rejection. The recently identified subset of non-circulating, tissue-resident memory $T$ cells $\left(T_{R M}\right)$ represents one important form of local immune responders within the grafts ${ }^{24}$. Another important contributor to the pathogenesis of chronic rejection is the formation of tertiary lymphoid organs (TLO) within the graft. TLOs represent important local immune response sites $^{25,26}$. The functions of $\mathrm{T}_{\mathrm{RM}}$ in graft rejection are not known. The mechanism of TLO formation in the graft is also not clear. The inability to distinguish resident from host circulating systemic responses after transplantation remains an obstacle to studying local immune responses. Retransplantation of a graft to a congenically disparate secondary recipient offers the ability to discern and specifically study the resident compartment of the primary recipient's immune response.

Re-transplantation of mouse heart allograft has been developed in our $\mathrm{lab}^{27}$ and by other investigators ${ }^{28}$. However, without treatment, heterotopic heart allografts are acutely rejected and are not life supporting ${ }^{1}$ thus minimizing the utility of retransplanting a heart in the study of chronic rejection. Till date, mouse kidney allograft re-transplantation has not been reported primarily due to difficulties in the secondary 
revascularization of the graft, re-establishment of the urinary tract continuity, and dissecting extensive adhesions during harvesting a transplanted kidney and ureter. We recently successfully established a mouse kidney re-transplantation model to investigate the role of $T_{R M}$ in kidney allograft rejection ${ }^{19}$. This detailed stepwise protocol will be of great value to nephrologists, immunologists, transplantation scientists, and microsurgeons interested in studying the local immune response.

\section{Overview of the protocol: advantages and limitations}

Re-transplantation of a mouse kidney allograft includes five stages: 1) primary donor graft harvest, 2) back-table preparation of the primary donor graft, 3) primary kidney transplantation, 4) secondary donor graft harvest from a primary recipient, and 5) re-transplantation of the secondary donor graft to a secondary recipient. We provide modifications to previously reported mouse kidney transplantation procedures $^{5,6,8}$ in Stage 1 and 3 to improve survival. Despite its importance, Stage 2 has never been as well described. Stages 4 and 5 are novel and described in detail in this protocol.

Similar to most reported protocols, we opted to intra-abdominally position the mouse kidney graft at the recipient's right flank instead of ectopically implanting it in the neck ${ }^{29}$. Positioning the kidney at the flank is the most physiological and clinically relevant way. The kidney can be positioned at either flank. However, since the recipient's inferior vena cava (IVC) is closer to the right side, using the right flank will cause less tension at the venous anastomosis site and hence offer a decreased risk of venous stricture and bleeding. Left donor kidneys were preferred owing to the greater length of the left donor renal vein 3,4 , $5,6,7,8,9,10,11,12,13,14$. However, the right donor kidneys can also be transplanted ${ }^{30}$. To re-establish arterial blood supply in the primary transplantation procedure, we used end-to-side vascular anastomosis by suturing a segment of donor $\mathrm{AO}$ to recipient's $\mathrm{AO}$. In contrast, the renal vein, a larger caliber vessel compared to the renal artery, was end to side anastomosed to the recipient's IVC. In the re-transplantation procedures, due to the difficulty in dissecting the renal vessels of a previously transplanted graft, we opted to utilize segments of the secondary donor/primary recipient's AO and IVC for end-to side vascular anastomosis to secondary recipient's AO and IVC, respectively. In this protocol, we introduce an interrupted "6-stitch" suturing style for arterial anastomosis. We gained $100 \%$ vascular anastomosis success rate (last consecutive 100 operations).

For ureteric reconstruction, due to the risk of bladder patch necrosis and subsequent urine leak, we preferred to use ureteric implantation with our modified drag-in technique. However, since ureteric implantation and bladder patch techniques have both advantages and disadvantages ${ }^{31,32}$, surgeons might opt to use either method depending on their experience. Details on how to effectively apply both methods are provided. Recipients underwent bilateral nephrectomy thus rendering the kidney grafts lifesupporting after transplantation. This allows surgical success to be assessed immediately after surgery by graft function and recipient's survival. Kidney function can be monitored by serum creatinine or blood urea nitrogen measurement. 
To note, re-transplantation of heart or kidney allografts in rats had been used previously to study the influences of early episodes after transplantation on the long-term graft outcome $33,34,35,36$. However, although technically demanding, mouse kidney re-transplantation provides unique advantages over rat models, or other large animal models, because the mouse gene background is well characterized, the diversity and availability of transgenic mice are greater, and the animals and related research reagents

are at a relatively lower price. Furthermore, the rejection of a mouse kidney allograft is slow ${ }^{37}$. This makes mouse kidney allograft re-transplantation a better model to study local immune responses, which need sufficient time to be established after transplantation ${ }^{19}$. The purpose of this protocol is to provide repeatable stepwise procedures for mouse kidney re-transplantation.

\section{Alternative Methods}

Some microsurgeons adopt techniques that involve anastomosing cuffs of donor $A O$ and IVC, similar to a carrel patch that is applied in clinical kidney transplantation, to the recipient $A O$ and IVC, respectively ${ }^{3}$ 38 . Others use a heel-and-toe $A O$ cuff for suture anastomosis ${ }^{12,39}$ and a cuff for non-suture renal vein connection ${ }^{10}$. These techniques are time consuming because the vascular patches/cuffs need to be prepared. Using segments of the donor AO and IVC instead of patches is a quicker ${ }^{4}$ and a more widely used method. The donor $A O$ segments can be superior renal ${ }^{4,6,9,30,40}$ or inferior renal ${ }^{5,8,30,31}$ based on individual preference and surgical needs. Running sutures is widely adopted for both arterial and venous anastomosis. However, interrupted suturing of the renal artery has a higher success rate ${ }^{31}$.

For urinary tract reconstruction, four approaches are available: 1) Anastomosing donor bladder patch to a cystectomy on the recipient bladder dome in one-layer $3,4,5,8,38$, 2) Suturing donor bladder patch to the recipient bladder dome in two-layers with ${ }^{7}$ or without ${ }^{13}$ anti-reflux bladder seromuscular tunnel (similar to the Lich-Greoir technique), 3) Dragging donor ureter distal end into the recipient bladder $6,9,11,30,31,40$, and 4) Pulling the distal part of the ureter including a small bladder patch $(2 \mathrm{~mm} \mathbb{E})$ into the recipient bladder $^{14}$. Given the small size of a mouse's ureter, using a donor bladder patch was previously considered as the only practical choice for re-establishment of the ureteric continuity in mouse kidney transplantation ${ }^{5}$. After Han et al. introduced the ureter-dragging-into-bladder technique of ureteric implantation in $1999^{6}$, a growing number of investigators started to recognize its simplicity, feasibility, and superiority $9,11,30,31,40$. The latter technique also facilitates kidney re-transplantation ${ }^{30}$ as described in this protocol.

\section{Reagents}

-Isoflurane, United States Pharmacopeia (USP) liquid for inhalation, $250 \mathrm{ml}$ (Piramal Healthcare, cat. no. NDC 66794-017-25)

-Oxygen, compressed, USP (Matheson tri-gas, cat.no. UN1072) 
·Heparin sodium injection, USP (1,000 USP units per ml; Fresenius Kabi, cat. no. 504031)

-Normal saline, $0.9 \%$ (wt/vol) sodium chloride injection, USP (Baxter Healthcare Corporation, cat. no. 2B1324X)

·DPBS, Dulbecco's phosphate buffered saline (Biowhittaker, cat.no.17-512F)

·Cefazolin for injection, USP (APP Pharmaceuticals,cat. no. NDC 63323-238-61)

·Buprenex, buprenorphine hydrochloride (Reckitt Benckiser Pharmaceuticals, cat. no. NDC 12496-0757-1)

.70\% Ethanol (Decon Labs, cat. no. 2716)

·PVP scrub solution (povidone-iodine 7.5\%; Medline, cat. no. NDC 12496-0757-1)

\section{Equipment}

EQUIPMENT

The authors prefer the listed materials and equipment solely based on personal experience. Investigators are advised to use their preferred equipment.

-Leica operating Microscope (Leica Microsystems Inc., cat.no. M651)

-Heated operating table, 290×200×65 mm, with electrical heating pad and cork pad (Vestavia Scientific, cat.no. 03-01)

-Thermal Cautery Unity (Geiger medical technologies, cat.no. 091500082)

·Isoflurane vaporizer (Summit Anesthesia Solutions, cat.no.22981)

-Infrared heating lamp (Cole-Parmer scientific experts, cat.no. EW-03057-01)

-Electric heating pad (MABIS/DMI Healthcare, cat.no. W-DMI619513)

-Nonwoven sponges (Mckesson, cat.no.944442000)

-Cotton tipped applicators, swab (Puritan, cat.no.868-WCS)

-Gelatin sponge (Harvard apparatus, cat.no. 59-9853)

·Powder free sterile latex surgical gloves (Protective Industrial Products Inc. cat.no. 100-3201) 
BBD $1 \mathrm{ml}$ TB Syringe, slip tip with 27-gauge $\times 1 / 2$ needle (BD, cat. no. 309623)

·BD PrecisionGlide needle, 27-gauge × 1/2 (BD, cat. no. 305109)

-3M Transpore Surgical Tape (Minnesota Mining and Manufacturing, cat.no.1527-2)

·Busse Hosp Disposables Sterile field, 18 in×26 in(Fisher scientific, cat.no.696)

·BD Falcon tissue culture dish, $100 \times 20$ mm (Corning, cat.no.353003)

-Insulated foam container without lid (for containing ice for preserving grafts)

-6-0 silk, spool ( George Tiemann \& Co, cat.no. 160-1215-6-0)

-10-0 nylon (AROSurgical Instruments Corporation, cat.no. TK-107038)

.6-0 nylon (Ethicon, cat.no. $667 \mathrm{G}$ )

-4-0 vicryl (Ethicon, cat.no.J488G-BX)

-9-0 vicryl (Ethicon, cat.no.TG140-8)

-Vannas spring scissors (Fine science tools, cat.no. 91501-09; Fig. 1d)

·Vannas-Tübingen spring scissors (Fine science tools, cat.no.15008-08; Fig. 1d)

·Corneal scissors (Codman, cat.no.54-6572; Fig. 1d)

Semken forceps (Roboz, cat.no. RS-5242; Fig. 1d)

·Dumont Dumostar tweezer, \#5 (Fisher scientific, cat.no. 50-241-57; Fig. 1d)

-Dumont forceps, \#3c (Dumont, cat.no.11231-20; Fig. 1d)

·Bulldog clamp (Shanghai medical Equipment Co., cat.no. W40130; Fig. 1d)

-Mosquito forceps (Shanghai medical Equipment Co., cat.no. W40340; Fig. 1e).

·Castroveijo needle holder (Roboz, cat.no. RS-6412; Fig. 1e)

-Micro forceps (Shanghai medical Equipment Co., cat.no. WA3080; Fig. 1e)

-Micro dissecting scissors (Roboz, cat.no.RS-5912; Fig. 1e)

-Abdominal wall retractors (Custom-made; Fig. 1e)

All microsurgical equipments must be sterile prior to utilization. Our instruments were autoclaved ( $>120$ ${ }^{\circ} \mathrm{C}$ for $30 \mathrm{~min}$ ). 


\section{MICE}

Authors recommend that all mice weigh 25-30 grams and are 8 to 12 weeks of age (The Jackson Laboratory).! CAUTION All experiments involving animals must be performed in accordance with national and institutional regulations. This protocol has been approved by the University of Pittsburgh and in accordance with criteria outlined in the Guide for the Care and Use of Laboratory Animals, a publication of the US National Institutes of Health.

Primary Donors: Any strain can be used. For our study, we used (BALB/c x B6)F1.mAct-OVA $\left(\mathrm{H}-2^{\mathrm{b} / \mathrm{d}}\right)$ mice.

Primary Recipient: A congenic strain that can host a tractable immune response to the graft hence forming a local response as well. B6.CD45.1 (B6.SJL-Ptprca Pepc ${ }^{\mathrm{b}} /$ BoyJ, Thy1.2, CD 45.1) (H-2 ${ }^{\mathrm{b}}$ ) congenic mice were used as recipients in our experimental model.

Secondary Donor: The primary recipient is then used as a secondary kidney donor to be re-transplanted to a secondary recipient.

Secondary Recipient: Depending on the research question different strains can be used. Since we were solely studying the local immune response, we considered mouse strains that lack secondary lymphoid organs including aly/aly and splenectomized B6.Itbr-/- mice or mice that lack an adaptive immune system such as B6.Rag-/-үc-/- mice. In the latter, lymphopenia induced proliferation might pose a caveat for studying local immunity. We opted for a splenectomized B6.Itbr-/-mouse.

\section{EQUIPMENT SETUP}

\section{Abdominal Wall Retractor (AWR) setup}

Straighten the second loop of two $3 \mathrm{~cm}$ paper clips (Fig. 1e). Next, using mosquito forceps, bend the first loop (wider) to make an 80-degree angle thus forming an abdominal hook. The third loop (narrower) is anchored to the cork part of the operating table using a 27-gauge needle. Each arm of the AWR is used to retract either left or right side of the abdominal wall.

\section{Procedure}

\section{Primary Donor Surgery OTIMING 20-30 min}

1 Anesthesia. Place the primary donor in an induction chamber connected to an isoflurane machine. Adjust the isoflurane vaporizer to a maximum of $5 \%$ with continuous oxygen flow of $1 \mathrm{~L} / \mathrm{min}$. Anesthesia 
induction is verified by observing a decrease in mouse respiratory rate down to 1 breath per second.

2| Skin preparation. Move the animal out of the induction chamber. Shave the abdominal skin entirely using an electric hair clipper. Alternatively, razor blades, depilatory creams or scissors can be used.

! CAUTION Make sure that the mouse is fully anesthetized to avoid accidental injuries to the limbs due to mouse's movements. Repeat step 1 if mouse is awake or awakening during skin preparation.

3| Surgical Setup. Drape the surgical table in a sterile fashion. Place the anesthetized mouse on the thermostatic operating table under the surgical microscope. Set the vaporizer at $1-1.5 \%$ isoflurane with continuous oxygen flow of $1 \mathrm{~L} / \mathrm{min}$ for maintaining the mouse under general anesthesia using continuous isoflurane inhalation through a mouse anesthesia mask. Tape all limbs to maintain the animal in the proper operative position on the operating table (Fig. 1a). Swab the abdominal wall with povidone-iodine first and then with $70 \%$ ethanol.

! CAUTION Maintaining animal body temperature by using a thermostatic operating table or a heating pad during surgery is very important, especially for the recipients. Hypothermia will decrease the respiratory rate of the animal during surgery, significantly prolong the recovery time, and increase the mortality risk of the recipients after surgery. We highly recommend using a thermostatic operating table with a sufficient cork area. Such a table is better for maintaining temperature, easier positioning, and anchoring the AWR during surgery.

4| Make a midline abdominal incision from the xiphoid process to the pubis. After making the incision, decrease isoflurane to $0.5-1 \%$ for maintenance of anesthesia.

$\triangle$ CRITICAL STEP It is critical to observe the respiration of the mouse during the operation to assess the depth of anesthesia. Adjust the isoflurane vaporizer to maintain a respiratory rate at approximately one breath per second. Deep anesthesia may lead to postoperative coma and death.

5|Using the AWR to retract the abdominal walls, expose the abdominal cavity. Anchor the retractors as specified in AWR setup section above (Fig. 1a). If the operating table lacks a cork component, corks or styrofoam squares can be attached onto the operating table. 
6|Make a sterile surgical wound drape by cutting a diamond hole $(2 \times 2 \mathrm{~cm})$ in the middle of a dry nonwoven sponge $(4 \times 4$ in). Cover the mouse with the drape as to only expose the abdominal surgical field (Fig. 1a-c).

7 Wrap the small and large intestines using a warm wet nonwoven sponge $(4 \times 2$ in $)$ with the assistance of a wet cotton swab and place them on the cranial right side of the abdominal cavity to efficiently expose the left kidney for the subsequent procurement (Fig. 1a).

8| Double suture ligate the left adrenal artery and vein together and transect between the sutures (Fig. 2a).

9| Suture ligate or cauterize the left gonadal artery and vein. Suture ligate the left lumbar vein merging into the left renal vein (RV) (Fig. 2b). There is no need to ligate the left lumbar vein if it drains into the inferior vena cava (IVC) instead of left RV (Fig. 3a).

$\triangle$ CRITICAL STEP Special attention should be given to protect the ureter's blood supply. Any damage to the ureter blood supply will increase the risk of ureter necrosis after surgery. The arrow in Fig. $\mathbf{3 b}$ shows the ureter accompanying vessels are carefully dissected and protected. To note, we use gonadal as a collective term for the internal spermatic and ovarian vessels in males and females, respectively.

10| Dissect the abdominal aorta $(\mathrm{AO})$ using the dumostar tweezers and micro forceps and place one segment of a 6-0 silk tie under the dissected portion of the AO between the levels of origin of left and right renal arteries (Fig. 2c).

! CAUTION Do not damage the lumbar veins draining into the IVC. Alternative AO levels where the silk tie is placed are used when the right renal artery or the superior mesenteric artery (SMA) origins are very close to the left renal artery origin. Under such circumstances, the silk tie should be placed above the level of the SMA origin and the ligations of right renal artery and SMA will be performed during the back-table procedures using 10-0 nylon.

11 Gently dissect the portion where the left RV drains into the IVC using the dumostar tweezers and micro forceps to separate it from the anterior surface of the AO. Create a space under the left RV that allows the tip of the closed arms of the micro forceps to completely pass beneath it (Fig. 3c). 
$\triangle$ CRITICAL STEP The pre-prepared space guarantees that the RV can be immediately transected once the $A O$ is ligated (step 13). This provides earlier graft perfusion and reduces graft ischemia reperfusion (IR) time.

12| Prepare $1 \mathrm{ml} 4^{\circ} \mathrm{C}$ cold $50 \mathrm{U} / \mathrm{ml}$ heparin saline in $1 \mathrm{ml}$ syringe with 27-gauge needle for graft perfusion.

$\triangle$ CRITICAL STEP To avoid a potential air embolus, remove air bubbles prior to the infusion.

13| Ligate the AO using the silk suture that has already been placed under the AO at step 10 (Fig. 2c). By stretching the scissor blade through the space made in step 11, immediately cut the left RV at the level where it drains into the IVC using the corneal scissors. This will serve as a perfusion outlet (Fig. 2d). Then, immediately start step 14 . After cutting the RV, the mouse will bleed out. Cutting the AO in step 17 will further bleed the mouse and accelerate cardiac arrest. At such time, the anesthesia machine can be turned off.

$\triangle$ CRITICAL STEP The right gonadal artery (Fig. 2i) will be cut in most mice while cutting the left RV and will be ligated during the back-table procedures. We do not recommend doing any dissection or ligation to the right gonadal artery branches before cutting the left $\mathrm{RV}$, because it is easier and quicker to perform this during the back-table procedures after the kidney graft is procured and will avoid any injuries to the vessels due to extra dissections at this step.

14 Clamp the AO approximately $1 \mathrm{~cm}$ under the origin of the left renal artery. Slightly lift the $\mathrm{AO}$ up using the dumostar tweezer. Slowly inject $4{ }^{\circ} \mathrm{C}$ of cold heparinized saline solution prepared in step 12 by puncturing the AO just above the clamp site (Fig. 2e). When blood from the donor kidney is flushed out and the kidney turns evenly pale, stop the perfusion. It usually requires $0.5-1 \mathrm{ml}$ of perfusion solution to achieve adequate perfusion of the graft.

15| Cut the AO $5 \mathrm{~mm}$ below the left renal artery origin from the AO using the corneal scissors (Fig. 2f).

16 Cut the IVC, connective tissue, and all lumbar arterial branches along the right side of the AO till the level of the silk ligation which has been already performed in step 13. 
$\triangle$ CRITICAL STEP Make sure not to cut the lumbar arterial branches (Fig. 2i) too close to the AO. Leave enough length for an easier ligation during the back-table procedures.

17| Cut the AO above the ligation which has been already performed in step 13 (Fig. 2g).

18|Use a dry nonwoven sponge $(4 \times 2$ in) to absorb the blood and perform a right cranial visceral rotation. Better visualization of the $\mathrm{AO}$ segment and renal pedicle will be achieved.

19| Separate the connective tissues between the aortic segment and renal artery from the posterior wall of the abdominal cavity.

20| Transect the left ureter at the distal end (Fig. 2h). Separate it from the surface of the psoas major muscle.

$\triangle$ CRITICAL STEP The ureter transection should be placed as distal as possible so that the ureter length is sufficient for re-transplantation.

21 Gently lift the donor kidney's lower pole and cut the lumbar vein at the distal part of the ligation (Fig. $\mathbf{2 b}$, step 9). Cut the connective tissue around the kidney, between the kidney and the posterior abdominal wall and the adrenal gland.

22| Harvest the kidney graft with its arterial, venous, and ureteral connections intact. Immediately place the organ in a container with $4{ }^{\circ} \mathrm{C}$ cold normal saline.

23|Euthanize then discard the primary donor according to institutional guidelines.

\section{Back-table preparation of the primary kidney graft OTIMING 3-5 min}

24| Prepare an icebox using a tissue culture dish $(10 \times 2 \mathrm{~cm})$ containing crushed ice. Put a piece of nonwoven sponge $\left(4 \times 4\right.$ in) in the $4{ }^{\circ} \mathrm{C}$ cold normal saline. Take the wet cold sponge out and stretch it out 
on the top of the tissue culture dish lid under the microscope. Take the donor kidney out of the cold saline and put it on the sponge. Examine the ends of $\mathrm{AO}$ and $\mathrm{RV}$ under 25x power microscope.

$\triangle$ CRITICAL STEP Careful examination of the AO and RV ends is needed to make sure they were evenly cut and hence will form adequate anastomoses.

25|Perfuse the organ through the $\mathrm{AO}$ using $0.2-0.5 \mathrm{ml} 4{ }^{\circ} \mathrm{C}$ cold heparin saline in a 27-gauge syringe to eliminate any residual blood inside the graft and vascular lumens. Ligate the right gonadal artery which originates from the anterior wall of the $A O$ and the lumbar arteries which originate from the right or posterior wall of the AO using 10-0 nylon (Fig. 2i). Ligate the right renal artery or SMA using 10-0 nylon if alternative $\mathrm{AO}$ ligating levels as described in step 10 are applied.

$\triangle$ CRITICAL STEP In our experience, most mice's right gonadal arteries originate from the AO anterior wall and are more cranial than the draining site of the left renal vein into the IVC. Also, almost $50 \%$ of the mice have 1-2 lumbar arteries originating from the right or posterior wall of the AO portion which need to be harvested as a segment attaching to the renal artery. These arterial branches are small and hard to expose for ligation before perfusion (Fig. 2i). We highly recommend that the ligations of these branches are performed during back-table preparation. These branches, if not ligated, will cause severe bleeding after reperfusion in the recipient which can be potentially fatal. We suggest ligating these small branches instead of cauterizing them to avoid thermal injuries to the AO. See Supplementary Video 1 for additional guidance on back-table preparation of donor graft.

26| Move the donor kidney back into the $4{ }^{\circ} \mathrm{C}$ cold normal saline immediately for preservation.

\section{Primary Recipient Surgery OTIMING 50-60 min}

27|Anesthesia and laparotomy. Similar to steps 1-6 in primary donor surgery, anesthetize the primary recipient, remove the abdominal hair, prep the skin, make a midline incision, retract the abdominal wall to expose the abdominal cavity, and cover the mouse with a sterile surgical wound drape.

28 Wrap the small and large intestines using a warm wet nonwoven sponge $(4 \times 2 \mathrm{in})$ with the assistance of a wet cotton swab and perform a left cranial visceral rotation to efficiently expose the right abdominal cavity and recipient IVC and AO (Fig. 1b). 
29| Slightly lift the right ureter using the dumostar tweezers and cut the ureter with a mono-polar fine-tip cautery. Ligate the native right renal artery and vein together using 6-0 silk tie and remove the right native kidney of primary recipient (Fig. 4a).

30| Use 6-0 silk to temporarily ligate the small lumbar arteries and veins that originate from and merge into the AO and IVC respectively to prepare a portion of the AO and IVC for anastomosis. Dissect and remove the fat and connective tissue that adhere to the anterior walls of the $\mathrm{AO}$ and IVC.

$\triangle$ CRITICAL STEP There is no need to separate the AO from the IVC. The ideal position for arterial and venous anastomosis is between the origin of left RV and the iliac bifurcation of $\mathrm{AO}$ (the anastomosis window). The left or right lumbar veins usually drain into the renal veins. If they drain into the IVC, temporarily ligating them at the IVC may offer better preparation of AO and IVC as ideal anastomosis sites.

$\triangle$ CRITICAL STEP Multiple temporary ligations might be needed to achieve an ideal AO anastomosis window, although usually 1-2 temporary ligations are sufficient. More than 2 temporary ligations are often used when there is a horizontal arterial branch originating from the $A O$ running across the anterior wall of the IVC. In this case, longer portions of AO and IVC need to be prepared for the anastomosis. In addition, the anastomosis site might need to be moved to the upper or lower part of the anastomosis window so that the arterial branches can be avoided. However, if there is no way to avoid them, the branches need to be ligated.

31|Place the first bull-dog clamp on the distal part of the IVC and AO using the micro forceps and mosquito forceps holding the bulldog (Fig. 5).

32| Place the second bull-dog clamp on the proximal part of the IVC and AO similar to step $\mathbf{3 1}$ (Fig. 5).

$\triangle$ CRITICAL STEP The distance between the two bull-dog clamps should not be less than $5 \mathrm{~mm}$. It is necessary to gently press the IVC anterior wall toward the cranial direction using the micro forceps before finally placing the second bull-dog clamp so that the IVC is not overly dilated. Over dilation of the IVC will affect the operative exposure of the AO. It is also critical to observe the distension of the IVC between the clamps after the second bull-dog clamp is placed. If the distention of the clamped portion of the IVC continues, this represents unclamped venous inflow to the IVC and the temporary ligations placed in step 30 should be examined and adjusted to prevent any blood flow into the clamped part of the IVC. If not prevented, this will severely affect the subsequent anastomosis procedure and can be fatal to the recipient. See Supplementary Video 2 for additional guidance on placing temporary ligations and bulldog clamps to prepare recipient AO and IVC for anastomosis. 
33 Make a stitch at the anterior wall of the AO using the micro forceps holding 10-0 needle and leave the needle on the $\mathrm{AO}$ wall. Create an arteriotomy at the anterior wall of the AO by cutting the anterior wall under the bottom of the needle that stays on the AO wall using the vannas spring scissors (Fig. 5a).? TROUBLE SHOOTING

$\triangle$ CRITICAL STEP The recipient aortic arteriotomy should match the lumen size of the graft donor AO. Cutting too much of the anterior wall of the recipient $A O$ will lead to a size mismatch between donor $A O$ lumen and recipient $\mathrm{AO}$ arteriotomy. This will also increase the risk of stricture at the arterial anastomosis site and is fatal to the recipient.

34|Flush the AO through the opening at the anterior wall immediately using warm non-heparinized normal saline in $1 \mathrm{ml}$ syringe with 27-gauge needle. Stop flushing when all blood and any clogs are flushed out of the portion of the $\mathrm{AO}$ between clamps and the portion turns completely pale. Usually, it requires $0.3-0.5 \mathrm{ml}$ saline to achieve an adequate flush of the $\mathrm{AO}$.

$\triangle$ CRITICAL STEP Do not touch the AO intima with the syringe needle during flushing. Any damage to AO intima will increase the risk of thrombogenesis at the arterial anastomosis site. While flushing, directed cranially, gently and repetitively squeeze the $\mathrm{AO}$ anterior wall caudal to the anastomosis site with micro forceps. It is unnecessary to flush caudally.

$\triangle$ CRITICAL STEP Use the non-heparinized normal saline instead of heparinized normal saline to flush the AO before and during the anastomosis procedures to avoid prolonged bleeding at the anastomosis site after releasing the bulldogs.

35 Gently place the primary donor kidney on the right flank side of the recipient abdominal cavity. Use the micro forceps to hold the 10-0 needle with nylon and end-to-side anastomose the donor AO at the prepared site of the recipient AO. The anastomosis is established using the "6-stitch" technique using interrupted sutures with 10-0 nylon (Fig. 4b). Briefly, make the first stay stitch at 12 o'clock and the second stay stitch at 6 o'clock to fix the donor AO to the opening of the recipient AO (Fig. 5b). Secondly, make the third and fourth stitches at 2 o'clock and 4 o'clock (Fig. 5c). Then, rotate the operating table by 180 degrees and gently flip the kidney graft over to the left side of the recipient abdominal cavity (Fig. 1c). Flush the anastomosis site with normal saline. Make the fifth and sixth stitches at 2 o'clock and 4 o'clock (8 o'clock and 10 o'clock, respectively, before table rotation) to finish the arterial anastomosis (Fig. 5d).

$\triangle$ CRITICAL STEP Make sure that full thickness passes of the suture needle including the vascular adventitia and the intima are achieved and no posterior $\mathrm{AO}$ wall is hooked when suturing the anterior wall 
of the $\mathrm{AO}$.

$\triangle$ CRITICAL STEP Examine the 10-0 needle prior to suturing the AO wall. Any damage of the tip of the needle will increase the perforating injury to the $\mathrm{AO}$ wall and the potential to carry the adventitia into the lumen of the AO. This increases the risk of arterial thrombogenesis at the anastomosis site.

$\triangle$ CRITICAL STEP Employ interrupted suturing ("6-stitch" method) instead of a continuous running suture for arterial anastomoses, which guarantees arterial potency without stenosis or thrombosis after unclamping the AO. We have found that using more than 6 stitches is unnecessary. See Supplementary Video 4 in re-transplantation for additional guidance on the "6-stitch" technique of arterial anastomosis, which works in both primary transplantation and re-transplantation procedures.

36| After finishing the arterial anastomosis, keep the recipient and the kidney graft in the same position for venous anastomosis. Use the Vannas-Tübingen spring scissors to make a venotomy at the anterior wall of the IVC with the assistance of micro forceps (Fig. 5e). The primary donor renal vein is end-to-side anastomosed to the recipient IVC using a continuous running suture with 10-0 nylon (Fig. 4c).? TROUBLE SHOOTING First, place two stay stitches at 12 o'clock and 6 o'clock, respectively, to fix the primary donor RV end to the anastomosis site of the IVC. Second, finish the anastomosis of posterior wall inside the IVC lumen from 6 o'clock to 12 o'clock and let the needle go out of the lumen at 12 o'clock (Fig. 5f-g). Last, finish the anastomosis of the anterior wall from 12 o'clock to 6 o'clock. Flush the IVC with warm normal saline to expel any air bubbles in the IVC before finishing the last stitch (Fig. $\mathbf{h}$ ).

$\triangle$ CRITICAL STEP Flush all air bubbles out of the IVC before finishing the venous anastomosis. Any air bubbles in the IVC will lead to air embolus and may be fatal.

37| Gently flip the kidney back to the right flank side of the recipient. Slightly press the arterial anastomosis site with a dry cotton swab and remove the caudal bulldog clamp first and then release the cranial clamp using the mosquito forceps. Keep the swab on the arterial anastomosis site with gentle pressure for 10 seconds to stop the bleeding then slowly remove.

$\triangle$ CRITICAL STEP Gelatin sponge can be applied to the anastomosis site for hemostasis.

38| Rotate the operating table again by 180 degrees so that the animal caudal end faces the surgeon.

$39 \mid$ Remove all temporary ligations placed in step 30. 
40|Double check the anastomosis sites of the AO and IVC, and the graft pedicle. Obtain hemostasis by using dry cotton swab, gelatin sponge, cautery, or ligation. Few recipients need the above measures and most bleeds can be resolved by applying gentle pressure with swab, or cautery. See Supplementary Video 5 in re-transplantation for additional guidance on venous anastomosis, releasing the bulldogs, achieving hemostasis at the anastomosis site, and removing the temporary ligations, which works in both primary transplantation (RV to IVC, $A O$ to $A O$ ) and re-transplantation (IVC to IVC, $A O$ to $A O$ ) procedures.

41 Separate the connective tissues and fats from the distal part of the ureter graft using the dumostar tweezers and Vannas-Tübingen spring scissors (Fig. 6a).

$\triangle$ CRITICAL STEP Do not dissect the ureter too much to avoid injuring the ureter's arterial supply. Examine the ureter under the microscope and make sure the blood vessels are intact. The distal ureteral part that lacks its arterial supply will be transected off later.

42| Ligate the end of the ureter graft with 10-0 nylon to let the ureter dilate (Fig. 6a).

$\triangle$ CRITICAL STEP The dilation of the ureter at this step will help avoid ureter torsion and twisting during the ureter to bladder implantation.

43| Puncture through the recipient bladder from the anterior wall to the posterior wall using the Dumostar tweezers with assistance of Dumont forceps holding the bladder top.

$\triangle$ CRITICAL STEP Do not use the needle to puncture the bladder. Puncturing with a blunt device (tweezers' tip) offers better healing of the bladder seromuscular layer and decreases the risk of a urine leak.

44| Hold the 10-0 nylon ligated at the end of the ureter with the Dumostar tweezers and slowly pull the ureter through the recipient bladder from the posterior wall to the anterior wall (Fig. 6b).

$\triangle$ CRITICAL STEP Do not grab the ureter directly. Guiding the ureter through the bladder by grabbing the 10-0 nylon pre-ligated at the ureter end will decrease injuries to the ureter. It also provides a chance to reguide the ureter through or out of the bladder before appropriately fixing the ureter onto the posterior wall of the bladder. 
45| Make two stitches using 10-0 nylon to fix the connective tissues that accompany the ureter to the posterior seromuscular wall of the recipient bladder (Fig. 4d, 6d).

$\triangle$ CRITICAL STEP Two symmetrically distributed stitches should be made exactly at the same level of the ureter. They should be placed at the edge of the opening in the posterior wall formed by puncturing the bladder with the tweezers (Fig. 6d).

46| Pull the ureter out of the anterior side of the recipient bladder using the 10-0 nylon ligature and cut the distal part of the ureter. Gently lift the anterior wall of the bladder and let the end of ureter graft retract spontaneously into the recipient bladder (Fig. 6c).? TROUBLE SHOOTING

47| Make a "figure of eight" stitch to close the hole at the anterior wall of the recipient bladder (Fig. 4e, $6 c)$.

An alternative approach to establish the primary donor ureter to primary recipient bladder connection is attaching donor ureter with a donor bladder patch to the recipient bladder dome. When using this technique, the whole donor bladder with posterior urethra and whole left donor ureter should be procured en bloc (Fig. 7).

$\triangle$ CRITICAL STEP For the alternative technique, make sure the donor bladder patch is adequately nourished (Fig.7a, $\mathbf{c}$ and d) otherwise (Fig.7b) techniques described in step 41-46 should be adopted. The donor bladder patch should be trimmed down to an adequate size. A small sized patch (Fig.7c-d) risks ureteric obstruction caused by inappropriately suturing the ureteral orifice on the donor patch. Larger patches need more blood supply and inadequate blood supply will cause patch necrosis after surgery. The opening at the recipient bladder dome should also be as small as possible. It can be dilated with micro forceps to the size that matches the donor bladder patch. If the donor bladder patch is too large (and not necrotic), it will likely cause urine retention due to neurogenic bladder, which is fatal. The 9-0 absorbable vicryl sutures are recommended for suturing the donor bladder patch to the recipient bladder dome. In our experience, the technique of suturing donor bladder patch to recipient bladder dome has no apparent advantages when compared with dragging the donor ureter into the recipient bladder as described above, especially when using Balb/c mice as donors. Balb/c donor bladder patch has high risk of necrosis after surgery likely due to insufficient blood supply (Fig.7b). We recommend using the ureteric implantation (ureter-drag-in) technique to establish ureter-bladder connection in this model. See Supplementary Video 6 in re-transplantation for additional guidance on the ureteric implantation procedure. 
48 Displace the small and large intestines to the right side of the recipient using a wet cotton swab. Cauterize the recipient left ureter (Fig. 4f). Ligate the recipient left renal artery and vein together using 6-0 silk (Fig. $\mathbf{4 g}$ ) and remove the native left kidney.

49| Restore the small and large intestines to the recipient abdominal cavity and carefully re-position them using a wet cotton swab.

50| Leave $0.5 \mathrm{ml}$ warm normal saline in the abdominal cavity and remove the wound drape and the retractors.

51 Close the abdominal muscular layer using continuous suturing with 6-0 prolene. Close the abdominal skin layer using continuous suturing with 4-0 vicryl.

\section{Primary recipient recovery TIMING 15-20 min}

52|Disinfect the surgical wound with 70\% ethanol. Administrate antibiotic cefazolin $100 \mathrm{mg} / \mathrm{kg}$ in $0.3 \mathrm{ml}$ saline and analgesic buprenex $0.1 \mathrm{mg} / \mathrm{kg}$ in $0.1 \mathrm{ml}$ saline subcutaneously. Tag the mouse ear for identification and move it into an isolated cage on the heating pad for recovery.

$\mathbf{5 3}$ Observe the recipient mouse every 5 min until it is fully awake. Recipient mouse recovers within 20 min. Mouse is given ad libitum access to water and an antibiotic (Trimethoprim 275 ppm, Sulfadiazine $1365 \mathrm{ppm}$ ) chow for three weeks after surgery. After three weeks, chow is switched to regular.

\section{Secondary Donor Surgery TIMING 40-60 min}

54 Anesthesia and laparotomy. Anesthetize the primary recipient mouse, clean the abdominal hair, prep the skin, make a midline incision, retract the abdominal wall to expose the abdominal cavity, and cover the mouse with pre-cut wound drape similar to steps 1-6 in primary donor surgery.

55| Wrap the small and large intestines and place them on the left side of the mouse to expose the kidney graft (Fig. 1b). 
56 | Use a fine-tip cautery to dissect the kidney and ureter grafts, the kidney graft's artery and vein, and the mouse's bladder, AO and IVC. Separate them from the adherent connective tissues, intestines, and liver tissues. Separate the kidney and ureter from the posterior wall of the abdominal cavity.? TROUBLE SHOOTING

57| Ligate and transect all lumbar arteries and veins that respectively originate from and drain into the $A O$ and IVC from $1 \mathrm{~cm}$ below and $0.5 \mathrm{~cm}$ above the graft anastomoses. Skeletonize this portion of AO and IVC together and separate them from the posterior abdominal wall (Fig. 8a). There is no need to separate the IVC from the AO.? TROUBLE SHOOTING

58 Ligate the AO and IVC together above the level of the graft anastomosis site using 6-0 silk. Make a venotomy on the IVC at the bifurcation level immediately to make an outlet for the perfusion solution. Slightly lift the AO by clamping it above the bifurcation level using dumostar tweezers. Slowly inject 50 $\mathrm{U} / \mathrm{ml}$ cold $4{ }^{\circ} \mathrm{C}$ heparin saline in $1 \mathrm{ml}$ syringe with a 27-gauge needle to perfuse the secondary donor kidney graft. The needle should be inserted slightly below $>1 \mathrm{~cm}$ where the primary donor kidney is anastomosed but above the AO clamp site (Fig. 8b-d). When the blood from the kidney graft is flushed out and the kidney graft turns evenly pale, stop the infusion. It usually requires $0.5-1 \mathrm{ml}$ of perfusion solution to achieve adequate perfusion of the graft.

59| Transect the AO and IVC together at the level of $0.5 \mathrm{~cm}$ below the anastomosis site of the kidney graft using corneal scissors (Fig.8e).

60| Transect the AO and IVC together above the silk ligation performed in step 58 (Fig. 8f).

61| Transect the ureter graft at its entry into the bladder (Fig. 8g). Immediately harvest the kidney graft with connections of whole ureter, renal vein, renal artery, primary donor $\mathrm{AO}$, and skeletonized secondary donor $\mathrm{AO}$ and IVC. Put them in $4{ }^{\circ} \mathrm{C}$ cold normal saline for preservation.

62| Euthanize the secondary donor according to institutional guidelines. 
See Supplementary Video 3 for additional guidance on procuring transplanted kidney graft for retransplantation.

\section{Secondary recipient surgery OTIMING 50-60 min}

63 Re-transplant the kidney graft from the secondary donor to the secondary recipient by repeating steps 27-51. In comparison to the primary recipient surgery, the differences in secondary recipient surgery for kidney graft re-transplantation include: 1) Instead of using the primary donor mouse's AO for anastomosis, the graft renal artery with a segment of the primary donor mouse's $A O$ and a segment of the primary recipient mouse's AO is end-to-side anastomosed to the secondary recipient mouse's AO (Fig. 9b) See Supplementary video 4 for additional guidance on arterial anastomosis during re-transplantation; 2 ) Instead of using renal vein for anastomosis, the graft renal vein with a segment of the primary recipient's IVC is end-to-side anastomosed to the IVC of the secondary recipient mouse (Fig. 9c). See Supplementary video 5 for additional guidance on venous anastomosis during re-transplantation; 3 ) In step 45, instead of 2 stitches, a total of 3-4 well-proportioned stitches are needed to fix the ureter graft onto the posterior wall of the secondary recipient mouse's bladder due to thickening of ureter graft's wall after primary transplantation (Fig. 9d, d-2). See Supplementary video 6 for additional guidance on establishing connection and fixation of the ureter graft to the bladder of the secondary recipient during retransplantation.? TROUBLE SHOOTING

\section{Secondary recipient recovery TIMING 15-20 min}

64|Secondary recipient mouse was treated similarly to the primary recipient mouse.

65|Secondary recipient mouse recovers similarly to the primary recipient mouse within 20 min after surgery.

\section{Microscope powers}

Step 1-7, 12, 23-24, 26-28, 38, 48-55, 62-65, procedures for animal skin prep, anesthesia induction, abdominal opening, wrapping intestines, restoring intestines, abdominal closure, etc. were performed with the naked eye.

Step 8-11, 13-22, 29-32, 39-48, 56-61, 63, dissection, vessel ligation, nephrectomy, graft perfusion, ureter connection, etc. were conducted under $16 \times$ magnification. 
Step 25, 33-37, 63, back-table preparation, arterial and venous anastomosis were carried out under $25 \mathrm{x}$ magnification.

\section{Troubleshooting}

\section{? TROUBLE SHOOTING}

Trouble shooting advice can be found in Table 1.

\section{Time Taken}

\section{Timing}

Step 1-23, primary Donor Surgery: 20-30 min

Step 24-26, Back-table preparation of the primary kidney graft: 3-5 min

Step 27-51, Primary Recipient Surgery: 50-60 min

Step 52 and 53, Primary recipient recovery: 15-20 min

Step 54-62, Secondary Donor Surgery: 40-60 min

Step 63, Secondary recipient surgery: 50-60 min

Step 64 and 65, Secondary recipient recovery: 15-20 min

\section{Anticipated Results}

The mouse kidney re-transplantation model has not been previously reported. We have achieved $>90 \%$ success (close to $100 \%$ for vascular anastomoses) in mouse primary kidney transplantation prior to employing mouse kidney re-transplantation. In total, 6 allogeneic (F1.OVA kidney to B6.CD45.1 to B6.ltbr-/-) and 2 syngeneic (B6 to B6 to B6) re-transplantations were completed. The syngeneic and allogeneic re-transplants were performed on $14 \mathrm{~d}$ and $35 \mathrm{~d}$, respectively, after primary kidney transplantation. The pilot allogeneic re-transplant recipient survived $11 \mathrm{~d}$ post re-transplant. 4 out of 5 subsequent allogeneic recipients survived until the set endpoint and were harvested 70 days post retransplantation. One recipient died at 21 days. 2 syngeneic recipients survived till set harvest date of 30 days after re-transplantation. All recipients' grafts were functional post re-transplant with normal serum creatinine of $<0.2 \mathrm{mg} / \mathrm{dl}$ at harvest or prior to unanticipated death. Hematoxylin and eosin-stained sections of syngeneic primary or re-transplanted kidney grafts obtained $30 \mathrm{~d}$ after primary transplantation or re-transplantation is shown in Figure 10. The syngeneic primary and secondary grafts' histological sections showed no pathology. 


\section{References}

1. Chong, A.S., Alegre, M.L., Miller, M.L. \& Fairchild, R.L. Lessons and limits of mouse models. Cold Spring Harb Perspect Med 3, a015495 (2013).

2. Tse, G.H., Hughes, J. \& Marson, L.P. Systematic review of mouse kidney transplantation. Transp/ Int 26, 1149-1160 (2013).

3. Skoskiewicz, M., Chase, C., Winn, H.J. \& Russell, P.S. Kidney transplants between mice of graded immunogenetic diversity. Transplant Proc 5, 721-725 (1973).

4. Kalina, S.L. \& Mottram, P.L. A microsurgical technique for renal transplantation in mice. Aust N Z J Surg 63, 213-216 (1993).

5. Zhang, Z. et al. Improved techniques for kidney transplantation in mice. Microsurgery 16, 103-109 (1995).

6. Han, W.R., Murray-Segal, L.J. \& Mottram, P.L. Modified technique for kidney transplantation in mice. Microsurgery 19, 272-274 (1999).

7. Martins, P.N. Technique of kidney transplantation in mice with anti-reflux urinary reconstruction. Int Braz J Uro/ 32, 713-718; discussion 719-720 (2006).

8. Wang, J.J., Hockenheimer, S., Bickerstaff, A.A. \& Hadley, G.A. Murine renal transplantation procedure. J Vis Exp (2009).

9. Rong, S., Lewis, A.G., Kunter, U., Haller, H. \& Gueler, F. A knotless technique for kidney transplantation in the mouse. J Transplant 2012, 127215 (2012). 
10. Chen, H., Zhang, Y., Zheng, D., Praseedom, R.K. \& Dong, J. Orthotopic kidney transplantation in mice: technique using cuff for renal vein anastomosis. PLoS One 8, e77278 (2013).

11. Tse, G.H. et al. Mouse kidney transplantation: models of allograft rejection. J Vis Exp, e52163 (2014).

12. Plenter, R., Jain, S., Ruller, C.M., Nydam, T.L. \& Jani, A.H. Murine Kidney Transplant Technique. J Vis Exp, e52848 (2015).

13. Wenwei, C., Yirong, Y., Stevens, K.M., Heger, M. \& Peng, X. Application of modified small bladder patch-to-bladder double-layer sutures to improve renal transplantation in mice. Eur Surg 49, 17-22 (2017).

14. Ge, X., Timsit, M.O. \& Tullius, S.G. A novel mouse renal transplant technique. Transp/ Int 24, e35-37 (2011).

15. Camirand, G. et al. Multiphoton intravital microscopy of the transplanted mouse kidney. Am J Transplant 11, 2067-2074 (2011).

16. Walch, J.M. et al. Cognate antigen directs CD8+ T cell migration to vascularized transplants. $J$ Clin Invest 123, 2663-2671 (2013).

17. Oberbarnscheidt, M.H. et al. Non-self recognition by monocytes initiates allograft rejection. $J$ Clin Invest 124, 3579-3589 (2014).

18. Zhuang, Q. et al. Graft-infiltrating host dendritic cells play a key role in organ transplant rejection. Nat Commun 7, 12623 (2016). 
19. Abou-Daya, K.I. et al. Resident memory T cells form during persistent antigen exposure leading to allograft rejection. Sci Immuno/ 6 (2021).

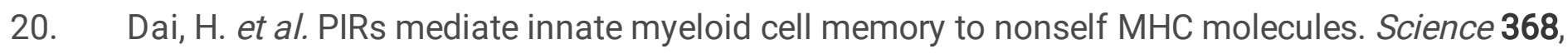
1122-1127 (2020).

21. Kirk, A.D., Knechtle, S.J., Larsen, C.P., Newell, K.A. \& Pearson, T.C. Miles to go. Am J Transplant 11, 1119-1120 (2011).

22. Oberbarnscheidt, M.H. \& Lakkis, F.G. Innate allorecognition. Immunol Rev 258, 145-149 (2014).

23. Lakkis, F.G. \& Li, X.C. Innate allorecognition by monocytic cells and its role in graft rejection. Am J Transplant 18, 289-292 (2018).

24. Snyder, M.E. et al. Generation and persistence of human tissue-resident memory $T$ cells in lung transplantation. Sci Immunol 4 (2019).

25. Baddoura, F.K. et al. Lymphoid neogenesis in murine cardiac allografts undergoing chronic rejection. Am J Transplant 5, 510-516 (2005).

26. Nasr, I.W. et al. Tertiary lymphoid tissues generate effector and memory T cells that lead to allograft rejection. Am J Transplant 7, 1071-1079 (2007).

27. Li, Q. et al. Technique for retransplanting heterotopic heart grafts in mice. Microsurgery 24, 465467 (2004).

28. Wang, K., Zhang, N. \& Li, H. Improved technique of mouse heterotopic heart graft retransplantation. Microsurgery 26, 200-202 (2006). 
29. Masutani, K., Zeng, G. \& Randhawa, P. Severe acute T cell and antibody-mediated rejection in ectopic kidney allografts with or without mouse polyomavirus infection. Am J Transplant 12, 3161-3162 (2012).

30. Tian, Y. et al. Kidney transplantation in mice using left and right kidney grafts. The Journal of surgical research 163, e91-97 (2010).

31. Huang, L.J., Reese, S. \& Djamali, A. Contributing factors to complications and surgical success in mouse kidney transplantation. Int Braz J Uro/ 38, 395-403; discussions 403-394 (2012).

32. Wang, Z.Z. et al. Optimized method for ureteric reconstruction in a mouse kidney transplant model. ANZ J Surg 84, 481-485 (2014).

33. Tullius, S.G., Hancock, W.W., Heemann, U., Azuma, H. \& Tilney, N.L. Reversibility of chronic renal allograft rejection. Critical effect of time after transplantation suggests both host immune dependent and independent phases of progressive injury. Transplantation 58, 93-99 (1994).

34. Tullius, S.G. et al. Prompt treatment of initial acute rejection episodes may improve long-term graft outcome. Transpl Int 11 Suppl 1, S3-4 (1998).

35. Tullius, S.G. et al. Contribution of early acute rejection episodes to chronic rejection in a rat kidney retransplantation model. Kidney Int 53, 465-472 (1998).

36. Minami, K. et al. C4d deposition and clearance in cardiac transplants correlates with alloantibody levels and rejection in rats. Am J Transplant 6, 923-932 (2006).

37. Hull, T.D., Benichou, G. \& Madsen, J.C. Why some organ allografts are tolerated better than others: new insights for an old question. Curr Opin Organ Transplant 24, 49-57 (2019). 
38. Russell, P.S., Chase, C.M., Colvin, R.B. \& Plate, J.M. Kidney transplants in mice. An analysis of the immune status of mice bearing long-term, $\mathrm{H}-2$ incompatible transplants. The Journal of experimental medicine 147, 1449-1468 (1978).

39. Plenter, R.J., Jain, S., Nydam, T.L. \& Jani, A.H. Revised Arterial Anastomosis for Improving Murine Kidney Transplant Outcomes. J. Invest. Surg. 28, 208-214 (2015).

40. Wang, M., Bai, J., Baumann, M. \& Heemann, U. New model for simultaneous heart and kidney transplantation in mice. Microsurgery 23, 164-168 (2003).

\section{Acknowledgements}

\section{Declaration}

All experiments involving animals were performed in accordance with national and institutional regulations. This protocol has been approved by the University of Pittsburgh institutional animal care and use committees and in accordance with criteria outlined in the Guide for the Care and Use of Laboratory Animals, a publication of the US National Institutes of Health.

\section{Acknowledgements}

This work was supported by NIH grants Al099465 and Al064343 to FGL, Al145881 to MHO, American Society of Nephrology Ben J. Lipps Research Fellowship and Stuart K. Patrick Grant for Transplant Innovation to KIA-D. RT was funded by T32 grant AI074490, F30 grant DK124925, and T32 grant GM008208 from the NIH.

\section{Author contributions}

DZ performed the surgery, analyzed histological data, and prepared the images. DZ, RT, and KIA-D postoperatively monitored animals. DZ and KIA-D prepared the videos and wrote the manuscript. JZ provided the schematic drawings. Z.C. designed the surgery. AA and AT provided experimental and intellectual input. KIA-D, MHO, and FGL conceived the model. All the authors critically revised the protocol. 


\section{Competing financial interests}

The authors declare no competing financial interests.

\section{Figures}

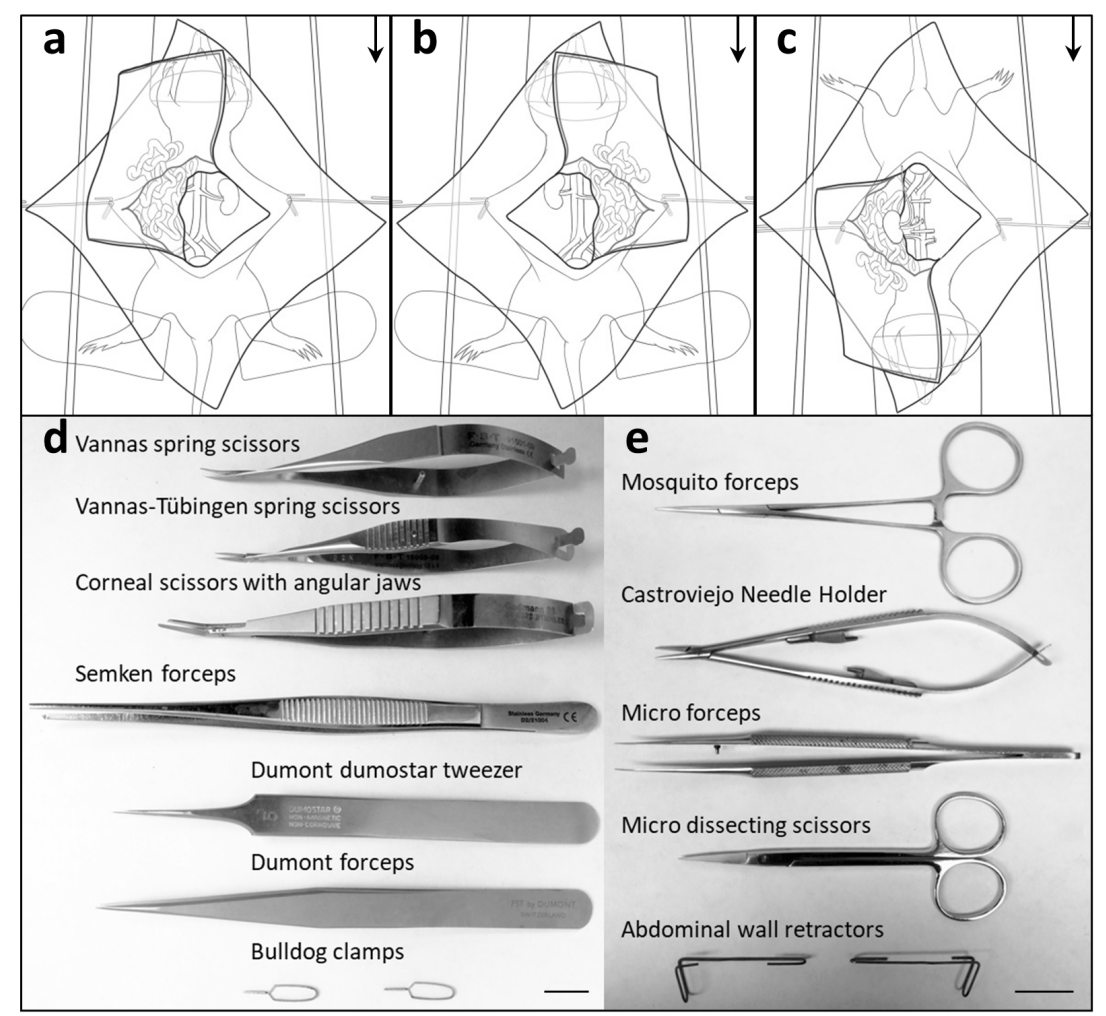

Figure 1 | Schematic drawings of operative positions and settings for mouse kidney transplantation or re-transplantation models and instruments used during surgery. (a) Donor position for primary kidney transplantation. (b) Recipient position for primary or secondary kidney transplantation during anastomosis of arterial anterior wall and donor position for secondary kidney transplantation. (c) Recipient position for primary or secondary kidney transplantation during anastomosis of arterial posterior wall and vein. (d-e) Instruments used throughout the protocol. (a-c) Arrows indicate surgeon's face side. Scale bars, $1 \mathrm{~cm}(\mathbf{d})$ and $2 \mathrm{~cm}(\mathbf{e})$. 


\section{Figure 1}

Schematic drawings of operative positions and settings for mouse kidney transplantation or retransplantation models and instruments used during surgery. (a) Donor position for primary kidney transplantation. (b) Recipient position for primary or secondary kidney transplantation during anastomosis of arterial anterior wall and donor position for secondary kidney transplantation. (c) Recipient position for primary or secondary kidney transplantation during anastomosis of arterial posterior wall and vein. (d-e) Instruments used throughout the protocol. (a-c) Arrows indicate surgeon's face side. Scale bars, $1 \mathrm{~cm}(\mathrm{~d})$ and $2 \mathrm{~cm}(\mathrm{e})$. 


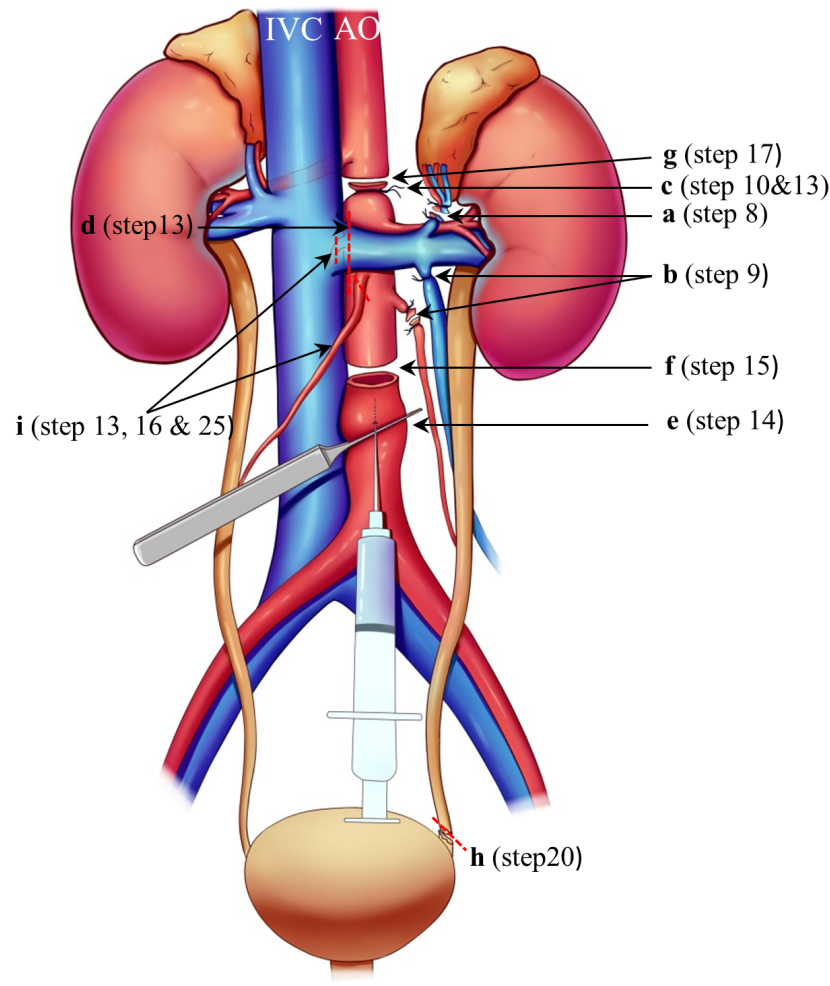

Figure 2 | Schematic drawing of mouse kidneys and demonstration of incisions for primary donor harvest. Labels indicate the following: (a) Ligate and cut adrenal vessels. (b) Ligate or cauterize the left gonadal artery and lumbar vein. (c) Place silk and ligate suprarenal AO. (d) Left renal vein incision line. (e) Clamp and pierce the AO to perfuse the left donor kidney. (f) Infrarenal AO incision level. (g) Suprarenal AO incision level. (h) Ureter incision line. (i) Cut and ligate right gonadal and lumbar arteries. AO, aorta. IVC, inferior vena cava.

\section{Figure 2}

Schematic drawing of mouse kidneys and demonstration of incisions for primary donor harvest. Labels indicate the following: (a) Ligate and cut adrenal vessels. (b) Ligate or cauterize the left gonadal artery and lumbar vein. (c) Place silk and ligate suprarenal AO. (d) Left renal vein incision line. (e) Clamp and pierce the AO to perfuse the left donor kidney. (f) Infrarenal AO incision level. (g) Suprarenal AO incision 
level. (h) Ureter incision line. (i) Cut and ligate right gonadal and lumbar arteries. AO, aorta. IVC, inferior vena cava.

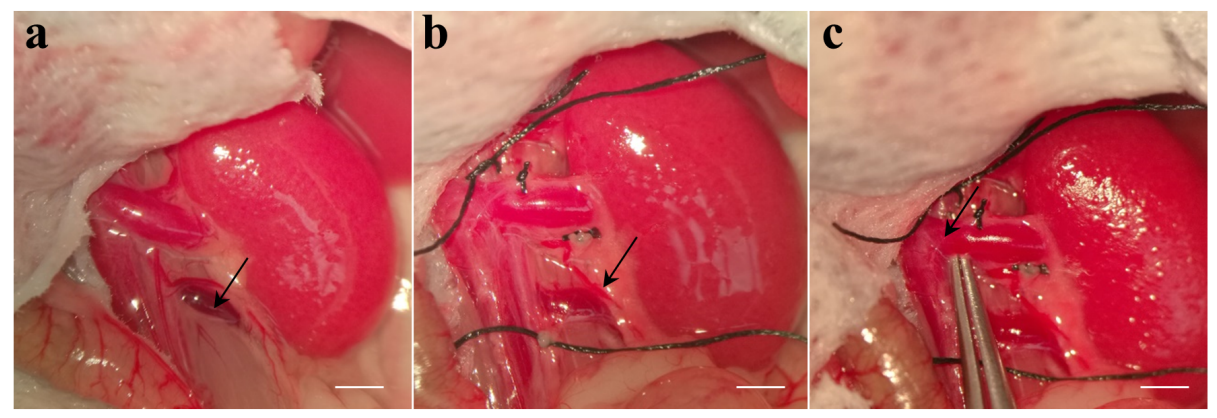

Figure 3 | Management of left lumbar vein, ureter accompanying vessels and left renal vein during primary donor kidney graft harvest. (a) There is no need to ligate the left lumbar vein (arrow) when it merges into the IVC instead of left RV. (b) In some cases, the ureter accompanying vessels (arrow) need to be carefully dissected and protected. (c) Make a space between the left RV (arrow) and anterior AO wall that allows the tip of closed arms of the micro forceps to totally go through under the left RV. AO, aorta. IVC, inferior vena cava. $\mathrm{RV}$, renal vein. Scale bars, $2 \mathrm{~mm}$. Experiments were performed under an institutional animal care and use committee-approved protocol.

\section{Figure 3}

Management of left lumbar vein, ureter accompanying vessels and left renal vein during primary donor kidney graft harvest. (a) There is no need to ligate the left lumbar vein (arrow) when it merges into the IVC instead of left RV. (b) In some cases, the ureter accompanying vessels (arrow) need to be carefully 


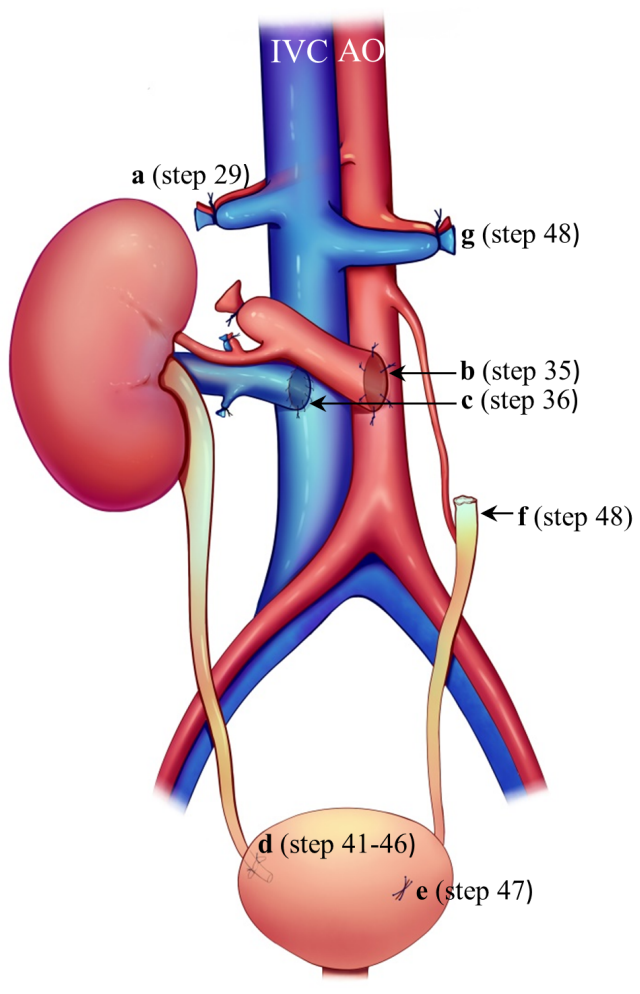

Figure 4 | Recipient schematic drawing of primary kidney transplantation. (a) Cauterize the right recipient ureter, ligate the native right renal artery and vein then remove the right native kidney. (b) Anastomose the donor AO to the recipient AO using "interrupted 6-stitch end-to-side" method. (c) Anastomose the donor renal vein to the recipient IVC using end-toside continuous suturing method. (d) Implant the donor ureter into the recipient bladder and secure it to the posterior wall of the recipient bladder. (e) Puncture closure at the anterior wall of the recipient bladder. (f-g) Cauterize the left recipient ureter, ligate the native left renal artery and vein then remove the left native kidney. (f) Recipient left native ureter stump. Similar right native ureter stump is not shown. Procedures are performed alphabetically. AO, aorta. IVC, inferior vena cava.

\section{Figure 4}


Recipient schematic drawing of primary kidney transplantation. (a) Cauterize the right recipient ureter, ligate the native right renal artery and vein then remove the right native kidney. (b) Anastomose the donor $\mathrm{AO}$ to the recipient $\mathrm{AO}$ using "interrupted 6-stitch end-to-side" method. (c) Anastomose the donor renal vein to the recipient IVC using end-to-side continuous suturing method. (d) Implant the donor ureter into the recipient bladder and secure it to the posterior wall of the recipient bladder. (e) Puncture closure at the anterior wall of the recipient bladder. $(f-g)$ Cauterize the left recipient ureter, ligate the native left renal artery and vein then remove the left native kidney. (f) Recipient left native ureter stump. Similar right native ureter stump is not shown. Procedures are performed alphabetically. AO, aorta. IVC, inferior vena cava. 

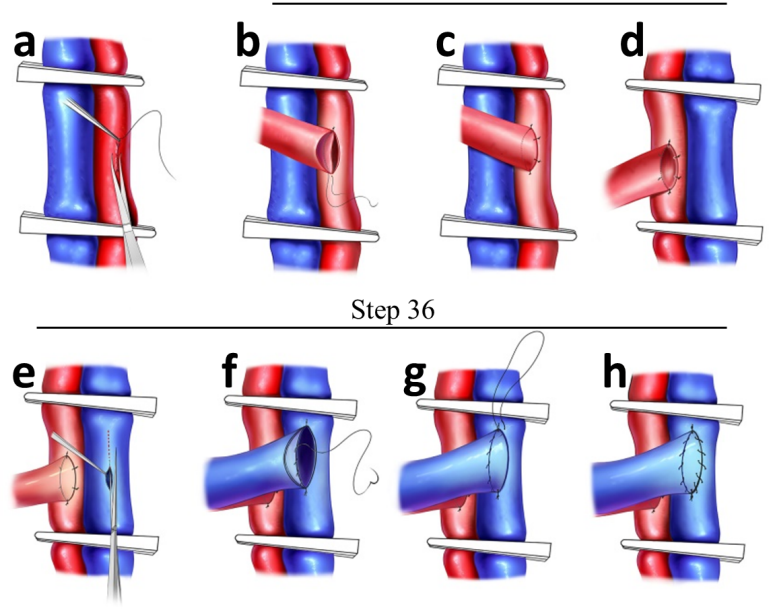

Figure 5 | Schematic drawings of vascular anastomosis. (a) Make a stitch at the anterior wall of the AO and leave the needle on the AO wall, then make an elliptic arteriotomy at the anterior wall of the $\mathrm{AO}$ by cutting the $\mathrm{AO}$ anterior wall under the bottom of the needle. (b) Make stay stitches at 12 o'clock and 6 o'clock to fix the donor AO to the arteriotomy of recipient AO. (c) Make 2 interrupted stitches at 2 o'clock and 4 o'clock to anastomose the anterior wall of the AO. (d) Rotate the operating table by 180 degree, flip the AO by 180 degree, then perform another 2 interrupted stitches at 2 o'clock and 4 o'clock (8 o'clock and 10 o'clock, respectively, before table rotation) to anastomose the posterior wall of the AO. (e) Make a venotomy at the anterior wall of the IVC. (f-g) Make 2 similar stay stitches as described in (b), then continuously suture the posterior wall of the vein inside the IVC lumen from 6 o'clock to 12 o'clock and let the needle go out of the lumen at 12 o'clock. (h) Continuously anastomose the anterior wall of the vein from 12 o'clock to 6 o'clock to finish anastomosis. a-c, the mouse tail is directed to the surgeon. $\mathbf{d - h}$, the mouse head is directed to the surgeon. AO (red), aorta. IVC (blue), inferior vena cava.

\section{Figure 5}

Schematic drawings of vascular anastomosis. (a) Make a stitch at the anterior wall of the $A O$ and leave the needle on the AO wall, then make an elliptic arteriotomy at the anterior wall of the AO by cutting the $\mathrm{AO}$ anterior wall under the bottom of the needle. (b) Make stay stitches at 12 o'clock and 6 o'clock to fix the donor $\mathrm{AO}$ to the arteriotomy of recipient AO. (c) Make 2 interrupted stitches at 2 o'clock and 4 o'clock to anastomose the anterior wall of the AO. (d) Rotate the operating table by 180 degree, flip the AO by 180 
degree, then perform another 2 interrupted stitches at 2 o'clock and 4 o'clock (8 o'clock and 10 o'clock, respectively, before table rotation) to anastomose the posterior wall of the AO. (e) Make a venotomy at the anterior wall of the IVC. (f-g) Make 2 similar stay stitches as described in (b), then continuously suture the posterior wall of the vein inside the IVC lumen from 6 o'clock to 12 o'clock and let the needle go out of the lumen at 12 o'clock. (h) Continuously anastomose the anterior wall of the vein from 12 o'clock to 6 o'clock to finish anastomosis. a-c, the mouse tail is directed to the surgeon. $d-h$, the mouse head is directed to the surgeon. AO (red), aorta. IVC (blue), inferior vena cava.
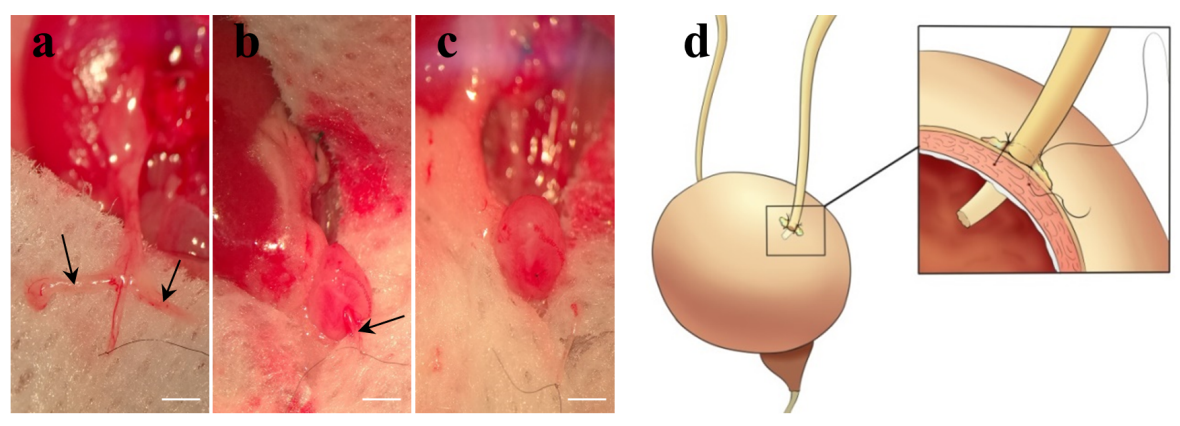

Figure 6 | Ureteric implantation. (a) Separate the connective tissues and fat (arrows) from the distal part of the ureter graft. Ligate the end of the ureter graft with 10-0 nylon to let the ureter dilate. (b) Puncture through the recipient bladder from the anterior wall to the posterior wall using the Dumostar tweezers. Hold the 10-0 nylon ligated at the end of the ureter to guide the ureter through the recipient bladder from the posterior wall to the anterior wall. Arrow indicates ureter end protruding from the hole formed by puncture on the anterior wall of the recipient bladder. (c) After fixing ureter graft to the posterior wall of the recipient bladder, trim the excess length of distal part of ureter from the outside of the recipient bladder. Close the puncture hole at the anterior wall of the bladder after the ureter end retracts into the bladder. Scale bars, $2.5 \mathrm{~mm}$. Experiments were performed under an institutional animal care and use committee-approved protocol. (d) Schematic drawing of fixing the ureter graft to the recipient bladder wall by 2 stitches of suturing the connective tissues accompanying the ureter to the seromuscular layer of the recipient bladder. 


\section{Figure 6}

Ureteric implantation. (a) Separate the connective tissues and fat (arrows) from the distal part of the ureter graft. Ligate the end of the ureter graft with 10-0 nylon to let the ureter dilate. (b) Puncture through the recipient bladder from the anterior wall to the posterior wall using the Dumostar tweezers. Hold the 10-0 nylon ligated at the end of the ureter to guide the ureter through the recipient bladder from the posterior wall to the anterior wall. Arrow indicates ureter end protruding from the hole formed by puncture on the anterior wall of the recipient bladder. (c) After fixing ureter graft to the posterior wall of the recipient bladder, trim the excess length of distal part of ureter from the outside of the recipient bladder. Close the puncture hole at the anterior wall of the bladder after the ureter end retracts into the bladder. Scale bars, $2.5 \mathrm{~mm}$. Experiments were performed under an institutional animal care and use committee-approved protocol. (d) Schematic drawing of fixing the ureter graft to the recipient bladder wall by 2 stitches of suturing the connective tissues accompanying the ureter to the seromuscular layer of the recipient bladder. 


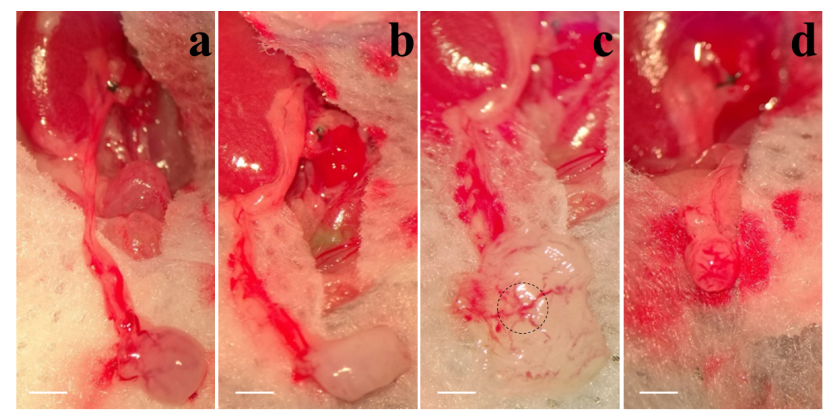

Figure 7 | Donor bladder patches after reperfusion. (a) Adequate donor bladder perfusion which is associated with lower risk of bladder patch necrosis. (b) Insufficient donor bladder perfusion posing a higher risk of bladder patch necrosis. (c) Dashed circle highlighting a bladder patch with adequate perfusion. At the center of the radially distributed vessels is the ureter's orifice, it should remain at the center after trimming the bladder patch. (d) A wellsized donor bladder patch with adequate perfusion. Scale bars, $2.5 \mathrm{~mm}$. Experiments were performed under an institutional animal care and use committee-approved protocol.

\section{Figure 7}

Donor bladder patches after reperfusion. (a) Adequate donor bladder perfusion which is associated with lower risk of bladder patch necrosis. (b) Insufficient donor bladder perfusion posing a higher risk of bladder patch necrosis. (c) Dashed circle highlighting a bladder patch with adequate perfusion. At the center of the radially distributed vessels is the ureter's orifice, it should remain at the center after trimming 

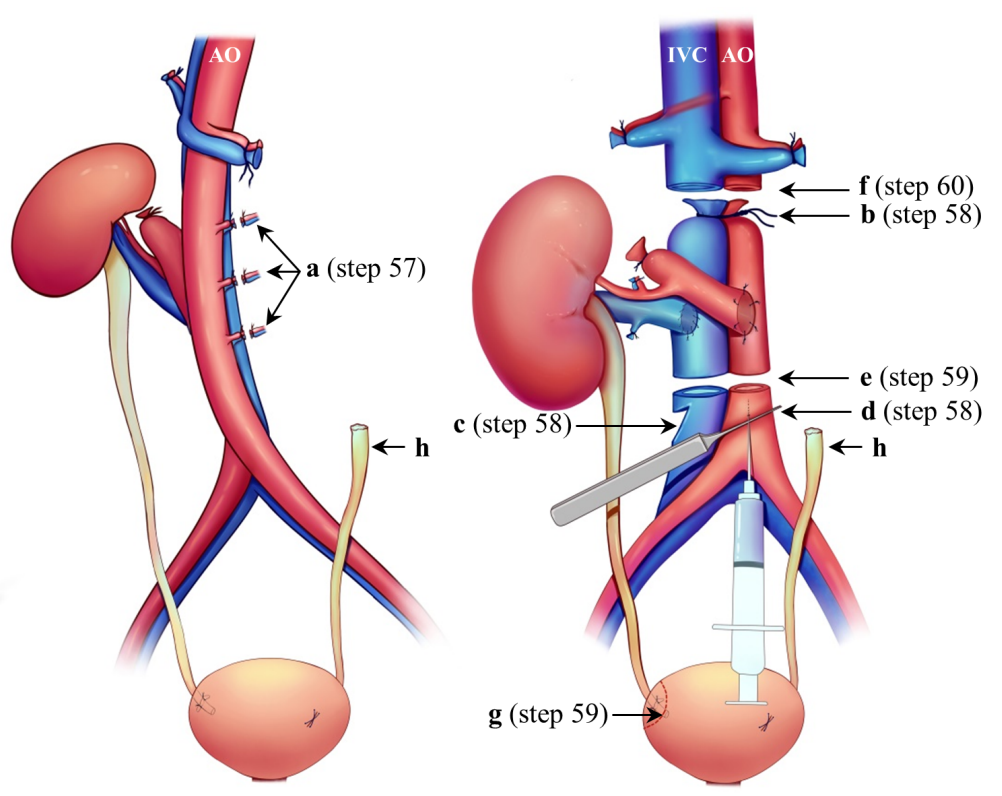

Figure 8 | Schematic drawings of secondary donor kidney graft harvest illustrating graft incisions. (a) Flip the AO to the primary recipient right side to expose and cut the lumbar arteries and veins after ligations. Skeletonize the portion of AO and IVC where the primary kidney graft was anastomosed. (b) Place a silk tie around the AO and IVC. (c) Make a venotomy on the IVC as an outlet for graft perfusion. (d) Clamp and puncture the AO to perfuse the secondary donor kidney. (e) AO and IVC incision level below the kidney graft. (f) AO and IVC incision level above the kidney graft. (g) Ureter graft incision line. (h) Secondary donor left native ureter stump. Similar right stump is not shown. Procedures are performed alphabetically. AO, aorta. IVC, inferior vena cava.

\section{Figure 8}

Schematic drawings of secondary donor kidney graft harvest illustrating graft incisions. (a) Flip the AO to the primary recipient right side to expose and cut the lumbar arteries and veins after ligations. Skeletonize the portion of $\mathrm{AO}$ and IVC where the primary kidney graft was anastomosed. (b) Place a silk tie around 
the AO and IVC. (c) Make a venotomy on the IVC as an outlet for graft perfusion. (d) Clamp and puncture the AO to perfuse the secondary donor kidney. (e) AO and IVC incision level below the kidney graft. (f) AO and IVC incision level above the kidney graft. (g) Ureter graft incision line. (h) Secondary donor left native ureter stump. Similar right stump is not shown. Procedures are performed alphabetically. AO, aorta. IVC, inferior vena cava.

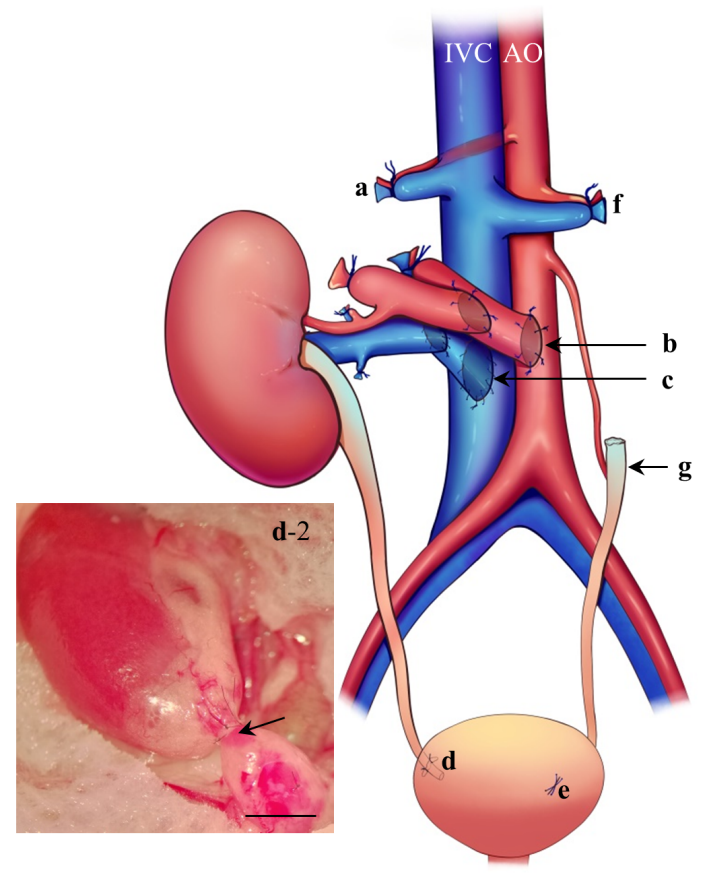

Figure 9 | Schematic drawing of mouse kidney re-transplantation. (a) Remove secondary recipient right native kidney. (b) The graft renal artery with a segment of the primary donor mouse's AO and a segment of the primary recipient mouse's AO is end-to-side anastomosed to the secondary recipient mouse's AO. (c) The graft renal vein with a segment of the primary recipient's IVC is end-to-side anastomosed to the IVC of the secondary recipient mouse. (d) A total of 3-4 well-proportioned stitches are applied to fix the ureter graft to the posterior wall of the secondary recipient mouse's bladder. d-2 shows a real image of 3 wellproportioned fixing stitches (arrow) for ureter re-transplantation. Scale bar, $3 \mathrm{~mm}$. (e) Close the puncture wound at the anterior wall of the secondary recipient bladder. (f) Remove the secondary recipient left native kidney. (g) Secondary recipient left native ureter stump. Similar right stump is not shown. Procedures are performed alphabetically. AO, aorta. IVC, inferior vena cava. 
Schematic drawing of mouse kidney re-transplantation. (a) Remove secondary recipient right native kidney. (b) The graft renal artery with a segment of the primary donor mouse's $A O$ and a segment of the primary recipient mouse's $A O$ is end-to-side anastomosed to the secondary recipient mouse's $A O$. (c) The graft renal vein with a segment of the primary recipient's IVC is end-to-side anastomosed to the IVC of the secondary recipient mouse. (d) A total of 3-4 well-proportioned stitches are applied to fix the ureter graft to the posterior wall of the secondary recipient mouse's bladder. $d-2$ shows a real image of 3 wellproportioned fixing stitches (arrow) for ureter re-transplantation. Scale bar, $3 \mathrm{~mm}$. (e) Close the puncture wound at the anterior wall of the secondary recipient bladder. ( $f$ ) Remove the secondary recipient left native kidney. (g) Secondary recipient left native ureter stump. Similar right stump is not shown. Procedures are performed alphabetically. AO, aorta. IVC, inferior vena cava. 


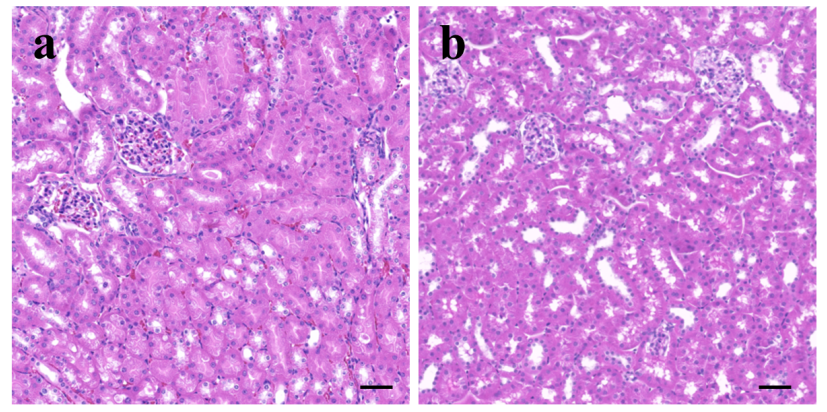

Figure 10 | Normal histology of syngeneic mouse kidney grafts $30 \mathrm{~d}$ after transplantation or re-transplantation. The tissue was fixed in $10 \%$ formalin for $24-48 \mathrm{~h}$ then stored in $70 \%$ ethanol. Hematoxylin and eosin staining was performed on paraffin-embedded tissue section. (a) Kidney graft $30 \mathrm{~d}$ after syngeneic primary kidney transplantation (C57BL/6 to C57BL/6). (b) Kidney graft $14 \mathrm{~d}$ after syngeneic primary kidney transplantation, then $30 \mathrm{~d}$ after syngeneic re-transplantation (C57BL/6 to C57BL/6 to C57BL/6). In syngeneic kidney graft re-transplantation, sections were histologically normal.Histopathology associated with rejection was not detected. Experiments were performed under an institutional animal care and use committee-approved protocol. Scale bars, $50 \mu \mathrm{m}$.

\section{Figure 10}

Normal histology of syngeneic mouse kidney grafts $30 \mathrm{~d}$ after transplantation or re-transplantation. The tissue was fixed in $10 \%$ formalin for $24-48 \mathrm{~h}$ then stored in $70 \%$ ethanol. Hematoxylin and eosin staining was performed on paraffin-embedded tissue section. (a) Kidney graft $30 \mathrm{~d}$ after syngeneic primary kidney transplantation (C57BL/6 to C57BL/6). (b) Kidney graft $14 \mathrm{~d}$ after syngeneic primary kidney transplantation, then $30 \mathrm{~d}$ after syngeneic re-transplantation (C57BL/6 to C57BL/6 to C57BL/6). In 
syngeneic kidney graft re-transplantation, sections were histologically normal.Histopathology associated with rejection was not detected. Experiments were performed under an institutional animal care and use committee-approved protocol. Scale bars, $50 \mu \mathrm{m}$.

\section{Supplementary Files}

This is a list of supplementary files associated with this preprint. Click to download.

- Table1.pdf

- SupplementaryVideo4.mov

- SupplementaryVideo5.mov

- SupplementaryVideo6.mov

- SupplementaryVideo1.mov

- SupplementaryVideo2.mov

- SupplementaryVideo3.mov 